\title{
Neural Network Based Hurdle Avoidance System for Smart Vehicles
}

\author{
Dr. B. Maruthi Shankar \\ Associate professor-ECE, Sri Shanmugha College of Engineering and Technology, Tamil Nadu \\ maruthishankar@gmail.com
}

\begin{tabular}{|c|c|}
\hline Article History & Abstract \\
\hline $\begin{array}{l}\text { Article Submission } \\
29 \text { August } 2019 \\
\text { Revised Submission } \\
22 \text { October } 2019 \\
\text { Article Accepted } \\
30 \text { November } 2019 \\
\text { Article Published } \\
31^{\text {st }} \text { December } 2019\end{array}$ & $\begin{array}{l}\text { The structure of a self-ruling vehicle dependent on neural sophisticated network for } \\
\text { route in obscure condition is proposed. The vehicle is equipped with an IR sensor for } \\
\text { obstacle separation estimation, a GPS collector for goal data and heading position, } \\
\text { L298 H-connect for driving the engines which runs the wheels; all interfaced to a } \\
\text { controller unit. The smaller scale controller forms the data gained from the sensor } \\
\text { and GPS to produce robot movement through neural based network. The neural } \\
\text { network running inside the small scale controller is a multi-layer feed-forward } \\
\text { network with back-engendering blunder calculation. The network is prepared } \\
\text { disconnected with tangent-sigmoid and positive direct estimate as enactment work for } \\
\text { neurons and is executed progressively with piecewise straight guess of tangent- } \\
\text { sigmoid capacity. The programming of the miniaturized scale controller is finished } \\
\text { by PIC C Compiler and the neural network is actualized utilizing MATLAB } \\
\text { programming. Results have shown that up to twenty neurons can be actualized in } \\
\text { shrouded layer with this method. The vehicle is tried with differing goal places in } \\
\text { open air situations containing fixed as well as moving obstructions and is found to } \\
\text { arrive at the set targets effectively and its yield exactness is about equivalent to that } \\
\text { of the normal precision. } \\
\text { Keywords: Autonomous Vehicles, Neural network, GPS, Feed forward network, } \\
\text { MATLAB. }\end{array}$ \\
\hline
\end{tabular}

\section{Introduction}

Self-governing robots with portable ability are finding their place in various applications. Some regular instances of these application fields are production line mechanization, administration application and unsafe situations, for example, perilous zones in atomic force stations, space investigation, material dealing with in medical clinic and security guarding. Route is the capacity of a versatile robot to arrive at the objective securely without human help. Hence the principle gives that should be tended to in versatile robot route are receptive obstruction evasion and target obtaining. Vision based detecting for self-governing route is an incredible and well known technique because of its capacity to give absolute data of condition which may not be accessible utilizing blends of different kinds of sensors [1].

The principle goal of this examination work is programmed route. The route task is partitioned into obstacle shirking and objective looking for errands. Obstacle shirking is accomplished with the assistance of infrared sensors. The range information from this sensor is taken care of to neural network running inside the small scale controller. To bring down the computational weight on miniaturized scale controller, neural network is actualized with piecewise straight guess of tangent-sigmoid and positive direct estimate actuation work for neurons [2]. Objective looking for conduct includes the information from GPS beneficiary which is prepared by smaller scale controller. Way point information for all stations is put away in the inner memory of the small scale controller. The smaller scale controller brings the ideal information and produces movement orders for the robot [3]. 
A natural neural network is made out of a gathering or gatherings of synthetically associated or practically related neurons. A solitary neuron might be associated with numerous different neurons and the all out number of neurons and associations in a network might be broad. Associations, called neurotransmitters, are normally framed from axons to dendrites. Aside from the electrical motioning, there are different types of flagging that emerge from synapse dissemination.

Man-made brainpower, intellectual demonstrating, and neural networks are data handling ideal models enlivened by the manner in which natural neural frameworks process information. Man-made reasoning and psychological demonstrating attempt to reenact a few properties of natural neural networks. In the man-made brainpower field, counterfeit neural networks have been applied effectively to discourse acknowledgment, picture examination and versatile control, so as to build programming (in PC and computer games) or independent robots [4].

\section{Existing techniques}

As of late there has been a developing enthusiasm for utilizing robots in exercises that are risky or cost restrictive for people to do. Such exercises incorporate military uses and space investigation. While automated equipment is regularly fit for being utilized in these sorts of circumstances, the capacity of human administrators to control robots in a powerful way is frequently restricted. This lack is regularly identified with the control interface of the robot and the degree of self-governance that control framework bears the human administrator [5]. This proposal portrays a robot control framework, called the safe/dangerous framework, which enables a human administrator to rapidly characterize how the framework can make the robot consequently perform deterrent shirking. This definition framework utilizes intuitive AI to guarantee that the hindrance evasion is both simple for a human administrator to utilize and can perform well in various situations. Beginning, true tests show that framework is powerful at programmed snag evasion.

Numerous analysts gave answer for the advancement of an affiliated neural network as an on-line calculation to prepare and control a putting out fires robot. Learning is remotely managed with encoded target activities. The robot gets fundamental route aptitudes just as the capacity to distinguish a fire and to smother it. The position, spatial recurrence and direction selectivity properties are accepted to have a significant job in visual discernment. The paper proposes a novel face portrayal and acknowledgment approach by investigating data mutually in picture space, scale and direction areas [6]. The face picture is first deteriorated into various scale and direction reactions by convolving multi-scale and multi-direction Gabor channels. Second, nearby double example examination is utilized to depict the neighboring relationship in picture space, yet in addition in various scale and direction reactions. In this way data from various spaces is investigated to give a novel face picture portrayal for acknowledgment. Neural Networks give huge advantages in face acknowledgment. They are effectively being utilized for such points of interest as finding already undetected examples, controlling gadgets dependent on criticism, and recognizing attributes in face acknowledgment. It improves the degree of precision contrasted and existing face acknowledgment strategies [7].

Another ongoing impediment evasion approach for portable robots has been created and executed in this paper. This methodology allows the location of obscure hindrances all the while with the directing of the versatile robot to evade impacts and progressing toward the objective. This methodology, entitled the Virtual Force Field, lies in the combination of two known ideas: Certainty Grids for obstruction portrayal, and Potential Fields for route. This blend is particularly appropriate for the convenience of mistaken sensor information, (for example, created by ultrasonic sensors) just as for sensor combination, and empowers constant movement of the robot ceaselessly before snags. This route calculation additionally considers the dynamic conduct of a quick versatile robot and tackles the "neighborhood least snare" issue. Trial results from a portable robot running at a most extreme speed of $0.78 \mathrm{~m} / \mathrm{sec}$ exhibit the intensity of the proposed calculation [8][9].

A multi-esteemed neuron (MVN) depends on the standards of numerous esteemed limit rationale over the field of the mind boggling numbers. The most significant properties of MVN are: the complex-esteemed loads, data sources and yield coded by the kth underlying foundations of solidarity and the actuation work, which maps the 
mind boggling plane into the unit circle. MVN learning is diminished to the development along the unit circle, it depends on a straightforward direct blunder remedy rule and it doesn't require a subsidiary [10]. It is demonstrated that utilizing a conventional design of multi-layer feed forward neural network (MLF) and the high usefulness of the multi-esteemed neuron, it is conceivable to acquire another amazing neural network. Its preparation doesn't require a subsidiary of the enactment capacity and its usefulness is higher than the usefulness of MLF containing a similar number of layers and neurons. These focal points of MLMVN are affirmed by testing utilizing equality n, two spirals and "sonar" benchmarks and the Mackey-Glass time arrangement expectation.

\section{Proposed Methodology}

In the proposed framework, plan of a self-governing vehicle dependent on basic micro-controller programming for route in obscure condition. The vehicle is furnished with four ultrasonic sensors for obstacle separation estimation, a wheel encoder for estimating separation voyaged, a compass for heading data, a GPS recipient for goal data, a GSM modem for changing goal place on run time and a non-unstable RAM for putting away way point information; all interfaced to an AT89C52 micro-controller. The micro-controller forms the data gained from the sensors and produces robot movement orders in like manner through coding of micro-controller.

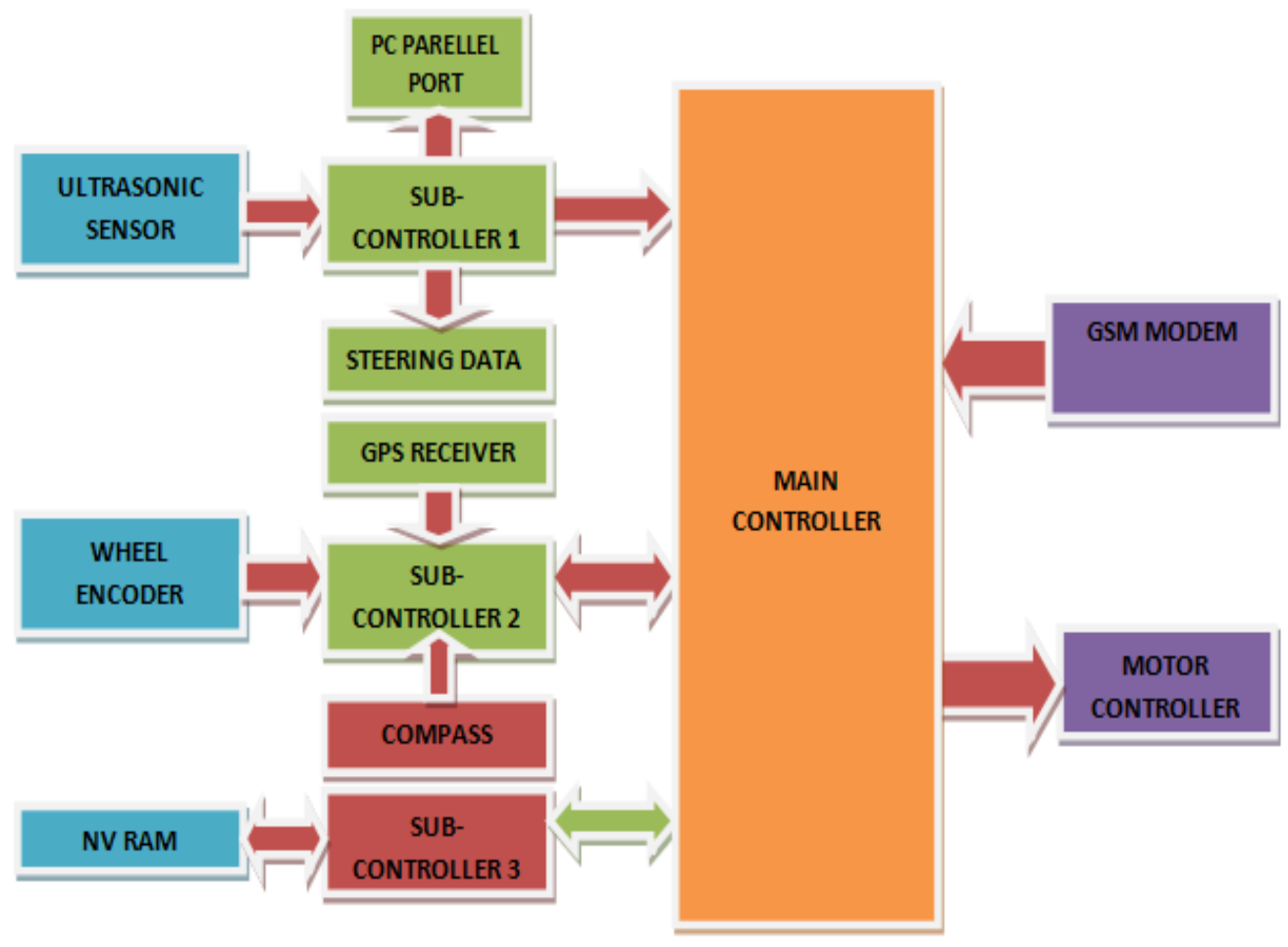

Fig 1: Hardware Setup of the Proposed System

To reduce the size of the circuit we eliminate certain components such as compass, wheel encoder, GSM modem, and AT89C52 microcontroller, non-volatile RAM by GPS receiver, L298 driver IC, high performance and high memory microcontroller PIC18F452 respectively. Programming through microcontroller is replaced by neural network programming. Neural network programming is used in non-linear system, through tangentsigmoid and positive linear function with piece-wise linear approximation of tangent oriented sigmoid function. The input layer of the neural network consists of the output from the sensors and latitude and longitude values of the GPS. This increases the speed of execution and decision making. This increases the accuracy of the output acquired. In the existing system the robot can move in forward, reverse, left, right direction. Since the sensors used in the existing are of low range it requires reverse motion when the obstacle is very near. But in the proposed system the range of the sensor is large hence no need for reverse motion. This eventually increases the efficiency of the robot's movement. 
In spite of the fact that the drawn out objective of the neural-arrange network remains the structure of selfgoverning machine insight, the primary current use of counterfeit neural systems is in the field of example acknowledgment. In the sub-field of information order, neural-organize strategies have been seen as valuable options in contrast to measurable procedures, for example, those which include relapse examination or likelihood thickness estimation. The likely utility of neural systems in the arrangement of multi-source satellitesymbolism databases has been perceived for well longer than 10 years, and today neural systems are a built up apparatus in the field of remote detecting.

\section{Simulation Results}

In arithmetic and registering, the Levenberg-Marquardt calculation (LMA), otherwise called the damped leastsquares (DLS) technique, is utilized to take care of non-straight least squares issues. These minimization issues emerge particularly in least squares bend fitting. The LMA presents between the Gauss-Newton count (GNA) and the methodology for edge plunge. The LMA is more impressive than the GNA, which infers that when in doubt it finds an answer whether or not it starts amazingly far away the last least. For conscious limits and reasonable starting limits, the LMA will as a rule be a piece more delayed than the GNA. LMA can in like manner be viewed as Gauss-Newton using a trust district approach.

The LMA is a notable curve fitting figuring used in various item applications for dealing with nonexclusive twist fitting issues. In any case, as for some fitting figuring, the LMA finds only a local least, which isn't generally the overall least. The Levenberg-Marquardt estimation has been applied to nonlinear speaks issues. A particular application is making computational models of oil supplies given the watched data. Like the semi Newton methods, the Levenberg-Marquardt computation was expected to advance toward second-demand getting ready speed without handling the Hessian structure. Exactly when the introduction work has the kind of a total of squares (as is normal in planning feed forward frameworks), The Jacobian structure can be prepared through a standard back inducing method that is impressively less astounding than calculating the Hessian organize.

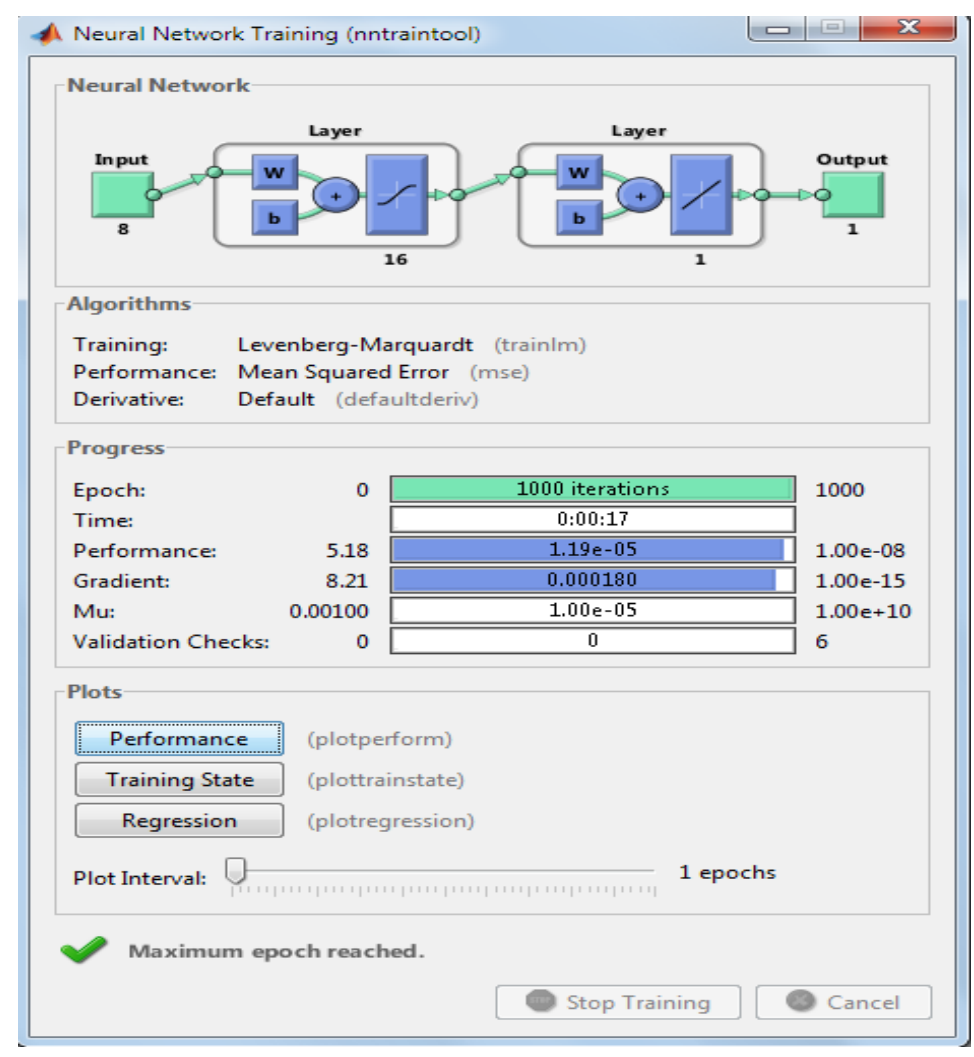

Fig 2: Training Window of the neural network

The Levenberg-Marquardt count uses this estimation to the Hessian organizes in the Newton-like update. Right when the scalar $\mu$ is zero, this is just Newton's method, using the evaluated Hessian arrange. When $\mu$ is 
tremendous, this becomes tendency drop with somewhat advance size. Newton's procedure is faster and dynamically accurate near a botch least, so the fact of the matter is to push toward Newton's methodology as quick as could be normal the situation being what it is. Along these lines, $\mu$ is lessened after each productive development (decline in execution work) and is extended exactly when a restrictive development would fabricate the show work. In this way, the display work is continually reduced at each pattern of the calculation.

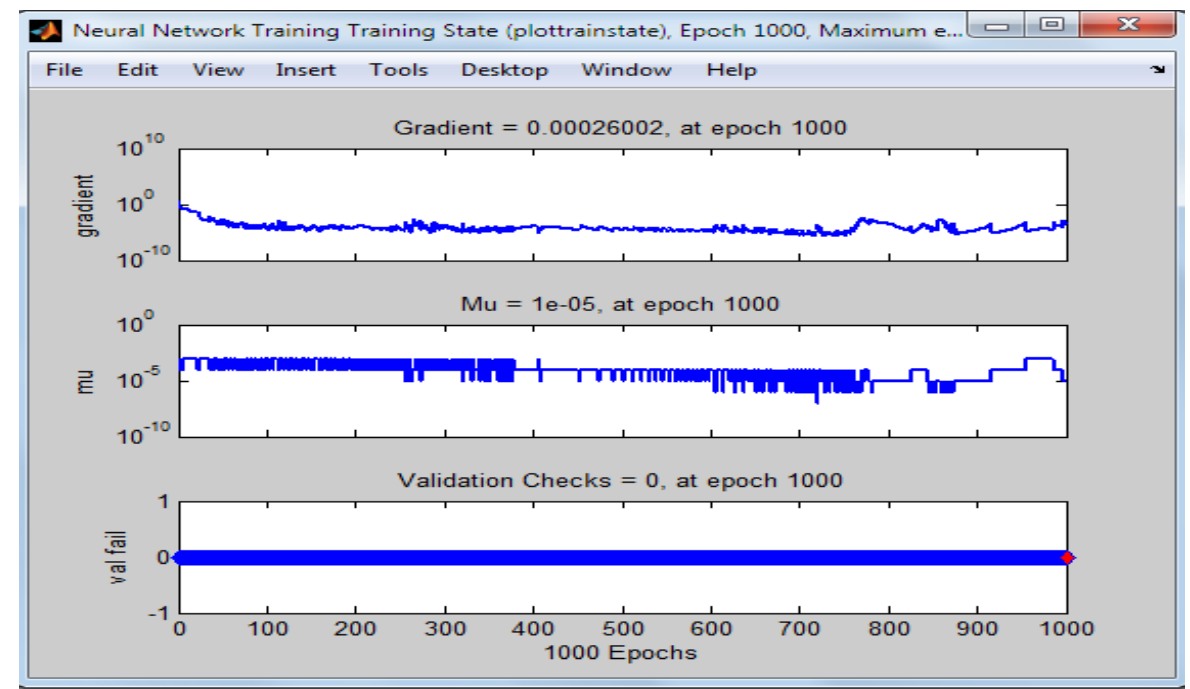

Fig 3: Simulation results showing gradient performance

This algorithm seems, by all accounts, to be the quickest technique for preparing moderate-sized feed-forward neural systems (up to a few hundred loads). It additionally has a proficient execution in MATLAB programming, in light of the fact that the arrangement of the network condition is a worked in work, so its characteristics become considerably increasingly articulated in a MATLAB domain. The Levenberg-Marquardt calculation is a basic, however hearty, strategy for approximating a capacity. The $\lambda$ damping factor is balanced at every cycle, and aides the streamlining procedure. On the off chance that decrease of $E$ is fast, a littler worth can be utilized, carrying the calculation closer to the Gauss-Newton calculation, though if emphasis gives lacking decrease in the remaining, $\lambda$ can be expanded, giving a bit nearer to the slope plunge bearing.

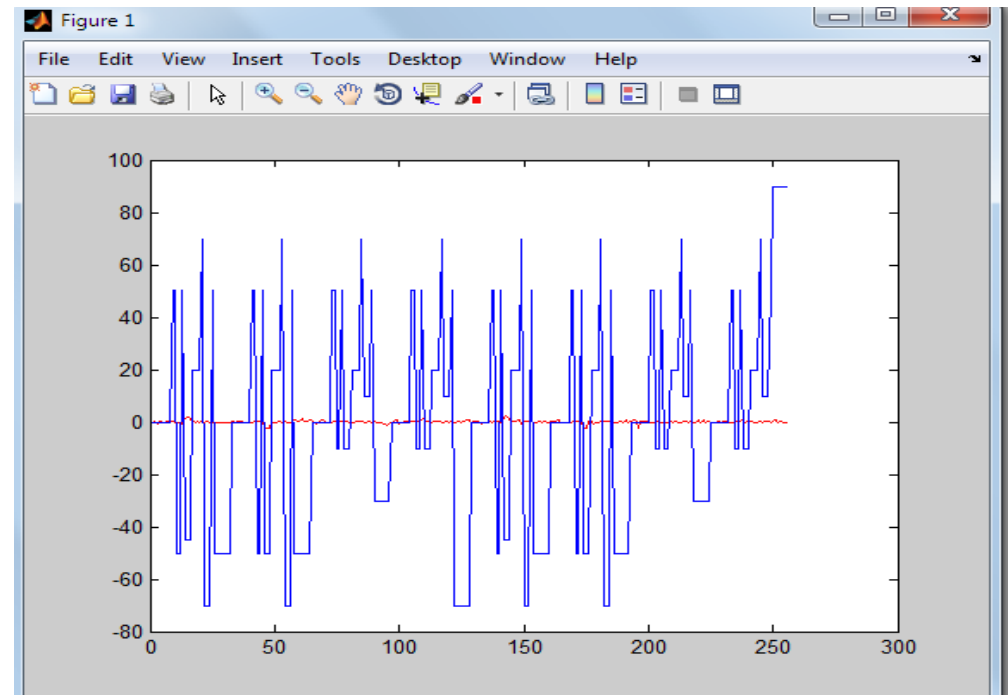

Fig 4: Simulation results showing neural network's performance

A sigmoid function is a limited differentiable genuine capacity that is characterized for all genuine information esteems and has a positive subsidiary at each point. Numerous regular procedures, including those of complex 
framework expectations to absorb information, show a movement from little beginnings that quickens and moves toward a peak after some time. At the point when an itemized depiction is deficient with regards to, a sigmoid capacity is regularly utilized. A sigmoid bend is delivered by a numerical capacity having a "S" shape. The preparation relapse plot of execution of the calculation in self-governing vehicle is appeared in figure 5 .

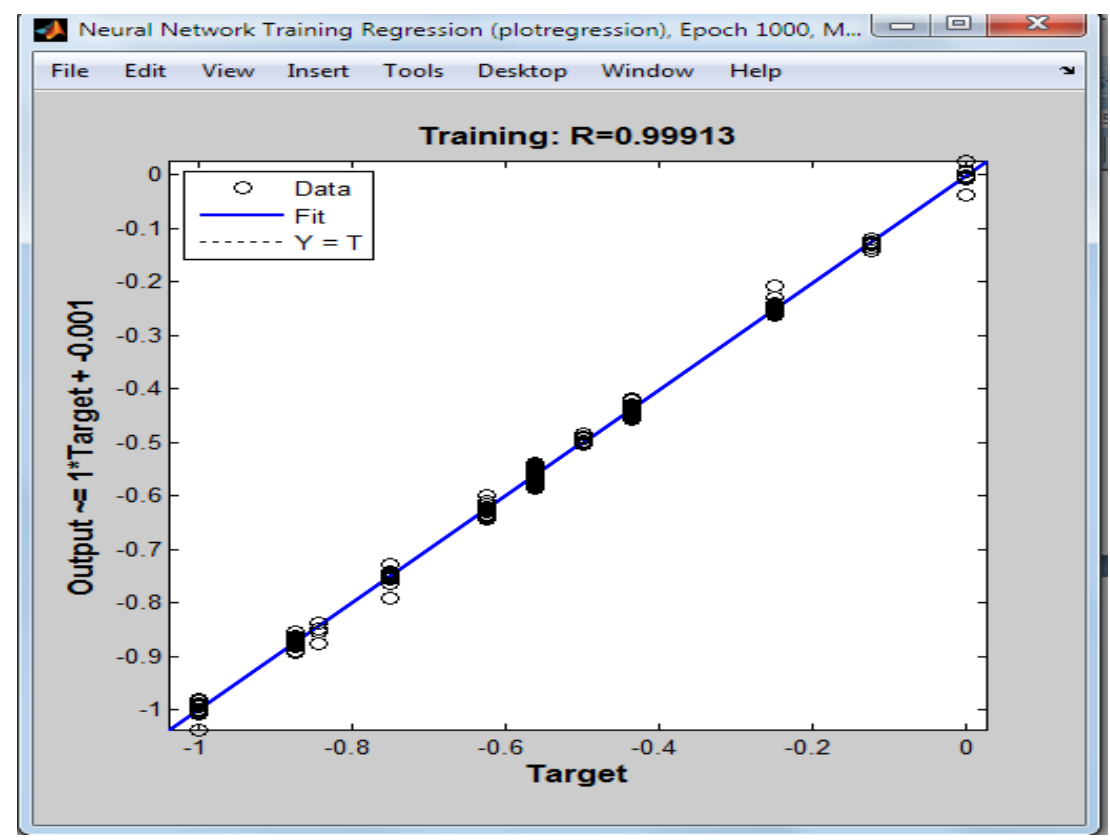

Fig 5: Simulation results showing plot regression of proposed system

\section{Conclusion}

Neural systems are appropriate for foreseeing time arrangement for the most part due to gaining just from models, with no compelling reason to include extra data that can bring more disarray than expectation impact. Neural systems can sum up and are impervious to clamor. Then again, it is commonly unrealistic to decide precisely what a neural system realized and it is likewise difficult to gauge conceivable forecast mistake. The recreated lattice yield of the neural system is actualized in equipment. To confront a non-straight condition it is executed with the assistance of implanted framework. Here the neural system which is actualized is a multilayer feed forward system with back engendering preparing calculation utilizing Levenberg-Marquardt advancement. The system is prepared disconnected with tangent-sigmoid and positive straight capacity as enactment work for neurons and is executed continuously with piecewise direct estimation of tangent-sigmoid capacity.

\section{References}

[1] Zhou, Y., Wilkins, D., \& Cook, R. P. (1994). Neural network for a fire-fighting robot. University of Mississippi.

[2] Borenstein, J., \& Koren, Y. (1989). Real-time obstacle avoidance for fast mobile robots. IEEE Transactions on systems, Man, and Cybernetics, 19(5), 1179-1187.

[3] Nielsen, L. (1988). Automated guidance of vehicles using vision and projective invariant marking. Automatica, 24(2), 135-148.

[4] Turner, J. M. (2007). Obstacle Avoidance and Path Traversal Using Interactive Machine Learning.

[5] Schepelmann, A., Snow, H. H., Hughes, B. E., Merat, F. L., Quinn, R. D., \& Green, J. M. (2009, November). Vision-based obstacle detection and avoidance for the cwru cutter autonomous lawnmower. In 2009 IEEE International Conference on Technologies for Practical Robot Applications (pp. 218-223). IEEE. 
[6] L. Cui, H. Jiang, B. B. Park, Y. Byon and J. Hu, "Impact of Automated Vehicle Eco-Approach on Human-Driven Vehicles," in IEEE Access, vol. 6, pp. 62128-62135, 2018, doi: 10.1109/ACCESS.2018.2874761.

[7] S. Cramer, I. Kaup and K. Siedersberger, "Comprehensibility and Perceptibility of Vehicle Pitch Motions as Feedback for the Driver During Partially Automated Driving," in IEEE Transactions on Intelligent Vehicles, vol. 4, no. 1, pp. 3-13, March 2019, doi: 10.1109/TIV.2018.2886691.

[8] G. Zhu, M. Yang, H. Li, B. Wang and C. Wang, "Curvature Map-Based Magnetic Guidance for Automated Vehicles in an Urban Environment," in IEEE Transactions on Intelligent Transportation Systems, vol. 17, no. 12, pp. 3541-3552, Dec. 2016, doi: 10.1109/TITS.2016.2557066.

[9] V. Stary, V. Krivanek and A. Stefek, "Optical detection methods for laser guided unmanned devices," in Journal of Communications and Networks, vol. 20, no. 5, pp. 464-472, Oct. 2018, doi: 10.1109/JCN.2018.000071.

[10] P. Yin, W. Li and Y. Duan, "Combinatorial inertial guidance system for an automated guided vehicle," 2018 IEEE 15th International Conference on Networking, Sensing and Control (ICNSC), Zhuhai, 2018, pp. 1-6, doi: 10.1109/ICNSC.2018.8361286. 